\title{
Temperature Stabilization of Group Index in Silicon Slotted Photonic Crystal Waveguides
}

\author{
Hassan Aghababaeian ${ }^{1 *}$, Mohammad-Hashem Vadjed-Samiei ${ }^{1}$, and Nosrat Granpayeh ${ }^{2}$ \\ ${ }^{I}$ Faculty of Electrical Engineering, Iran University of Science and Technology, Tehran, Iran \\ ${ }^{2}$ Faculty of Electrical and Computer Engineering, K. N. Toosi University of Technology, Tehran, Iran
}

(Received August 10, 2011 : revised October 10, 2011 : accepted October 25, 2011)

\begin{abstract}
In this paper, we have proposed a principle to design wideband, low dispersion and temperature stabilized slow light structure in slotted photonic crystal waveguide (SPCW). The infiltration of the silicon photonic crystal with polymer will enhance the slow light and increase the group index, whereas the different signs of thermo-optic coefficients of polymer and silicon make the proposed structure stable on temperature variation over $60^{\circ} \mathrm{C}$ and improves the group index-bandwidth products of the designed structure. The SPCW structure is modified to maximize the slow light effect and minimize the dependence of the group index and hence the group velocity dispersion to temperature.
\end{abstract}
Keywords : Slow light, Slotted photonic crystal waveguide, Dispersion, Temperature independent, Photonic devices
OCIS codes : (230.0230) Optical devices; (130.3120) Integrated optics devices; (260.2030) Dispersion; (120.6810) Thermal effects

\section{INTRODUCTION}

The slow light (SL) in photonic crystal waveguides (PCW) has been receiving interest during the past decade [1-4]. It enhances the light-matter interaction which reduces either the power or the physical length required to observe the same linear and nonlinear effects compared to the fast light regime [5]. In the slow light regime, the optical signal is compressed and hence the pulse amplitude is increased, which causes the enhancement of the linear and nonlinear effects in the structure. Linear effects such as gain, thermo-optic, and electro-optic interactions are scaled with the slow down factor, whereas the nonlinear effects may scale with its square $[2,6]$.

It is possible to design slow-light structures to modify dispersion diagrams to obtain high slow down factor and low group velocity dispersion (GVD). These structures have been recently proposed through engineering the geometry of the PCW, chirping the waveguide properties [2, 3], modifying the waveguide width [7], changing the hole size [3], and reshaping the two innermost rows of the holes in W1-PCW $[2,8]$. Since disordering limits the accessible group index and bandwidth [3], each method requires high precision fabrication technologies.

Light confinement and dispersion control have been studied in the silicon based slotted PCW in recent years [9-11]. The Electro-Optic (E-O) or nonlinear material injection to the slot region of the slotted PCW results in the light confinement and tuneability of the devices. The field enhancement in an E-O-polymer filled slotted waveguide [10] and the high speed modulation in photonic crystal (PC) Mach-Zehnder geometry have been studied [9, 11]. Also, slow light tuning in slotted PCW has been numerically demonstrated [12]. But the common problem in silicon photonic devices is the temperature dependence of the optical properties due to the thermo-optic effect of silicon [13, 14]. This effect has not been taken into account in the previous research on the slow light structures, whereas it has had a considerable effect on the optical properties of the designed structure. As an example only a $38^{\circ} \mathrm{C}$ temperature variation corresponds to variation of $6.8 \times 10^{-3}$ in refractive index which is enough to perform switching in some PC switches $[15,16]$. Therefore, to prohibit undesired change of transmission behaviour of the device caused by temperature variation, accurate temperature control is necessary, thus increasing the size and cost of the devices.

\footnotetext{
*Corresponding author: aghababaeian@iust.ac.ir

Color versions of one or more of the figures in this paper are available online.
} 
In this paper, we have proposed a potential solution to improve the design of silicon based slow light optical devices. In our silicon based slotted PCW design, we have modified the two innermost rows of the holes. The slot and the two innermost rows are infiltrated with polymer. These modifications change the dispersion of the guided modes in PCW, generate and enhance the slow light over a wideband with very low GVD. However, since the thermal dependence of refractive indices of silicon and polymer are typically opposite, the thermo-optic effect of the polymer can minimize or even eliminate the temperature dependence of the device for a desired operating range. We show that the proposed structure will be stable for temperature variation of up to $60^{\circ} \mathrm{C}$. Also the infiltration of the structure can be done after implementation of the main silicon structure, hence the normal fabrication precision is enough to obtain a structure with high group index-bandwidth product.

This paper is organized in four sections. The design of the slow light slotted PCW and the modified structure parameters to maximize broadband and low group velocity dispersion will be discussed in next section. The design, performance and thermal stability of the proposed structure are studied in section 3. The paper will be concluded in section 4.

\section{DESIGN AND SIMULATION}

The Planar slotted PCW (SPCW) can be implemented by replacing a row of air holes in the triangular lattice PC with a narrow slot, as displayed in FIG. 1. Since the two innermost rows of holes have the most important impact on the dispersion diagram $[1,8,17]$, these two rows are modified. The slot and the two innermost rows are infiltrated with polymer.

Two dimensional numerical calculations of the band

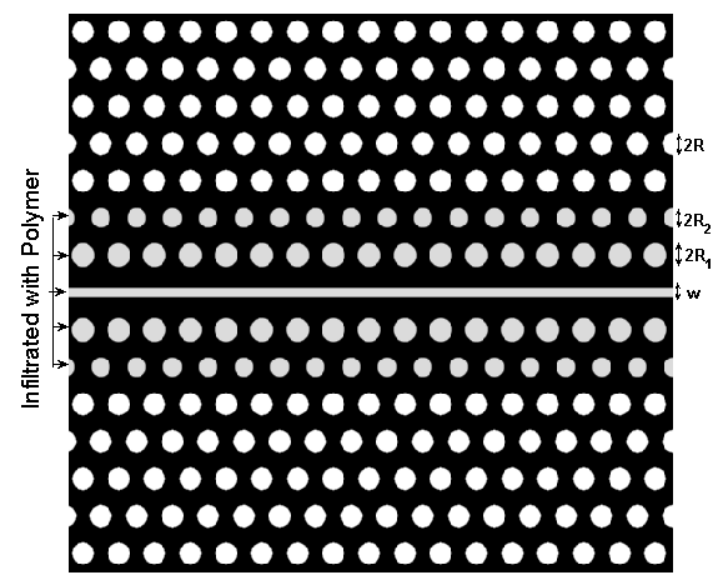

FIG. 1. Geometry of the slotted photonic crystal waveguide. The two innermost rows of holes of the PCW are modified. The slot and the two innermost rows of the holes are infiltrated with polymer. diagrams are performed by the plane wave expansion method (PWEM) and by selecting an appropriate supercell using the MIT photonic band (MPB) package [18]. Dispersion diagram of the TE mode in the proposed structure is depicted in FIG. 2a, which shows the PBG and single mode range of the structure. Related group index $\left(n_{g}=d k / d \omega\right)$ and GVD $\left(\beta_{2}=\frac{d^{2} k}{d \omega^{2}}=\frac{1}{c} \frac{d n_{g}}{d \omega}\right)$ are shown in FIG. $2 b$. The geometry parameters are considered as: silicon refractive index of $n_{s i}$ at the wavelength of $1.55 \mu \mathrm{m}$, lattice constant of $a=390 \mathrm{~nm}$, holes radii of $\mathrm{R}=0.3 a$ and the slot width of $\mathrm{W}=0.26 a$. In this work, we propose to use LFR-series low-loss and low-

birefringent polymers as infiltrating materials. These can be supplied by Chem Optics, Inc. The refractive index of LFR material is about 1.4 at wavelength of $1.55 \mu \mathrm{m}[22,23]$.

To flatten the dispersion diagram of the fundamental mode in a desired frequency, and to maximize the slow light over a wide bandwidth, the radii of the first and the second innermost rows of the holes are reduced and increased, respectively.

The dependence of the dispersion diagram on the diameter
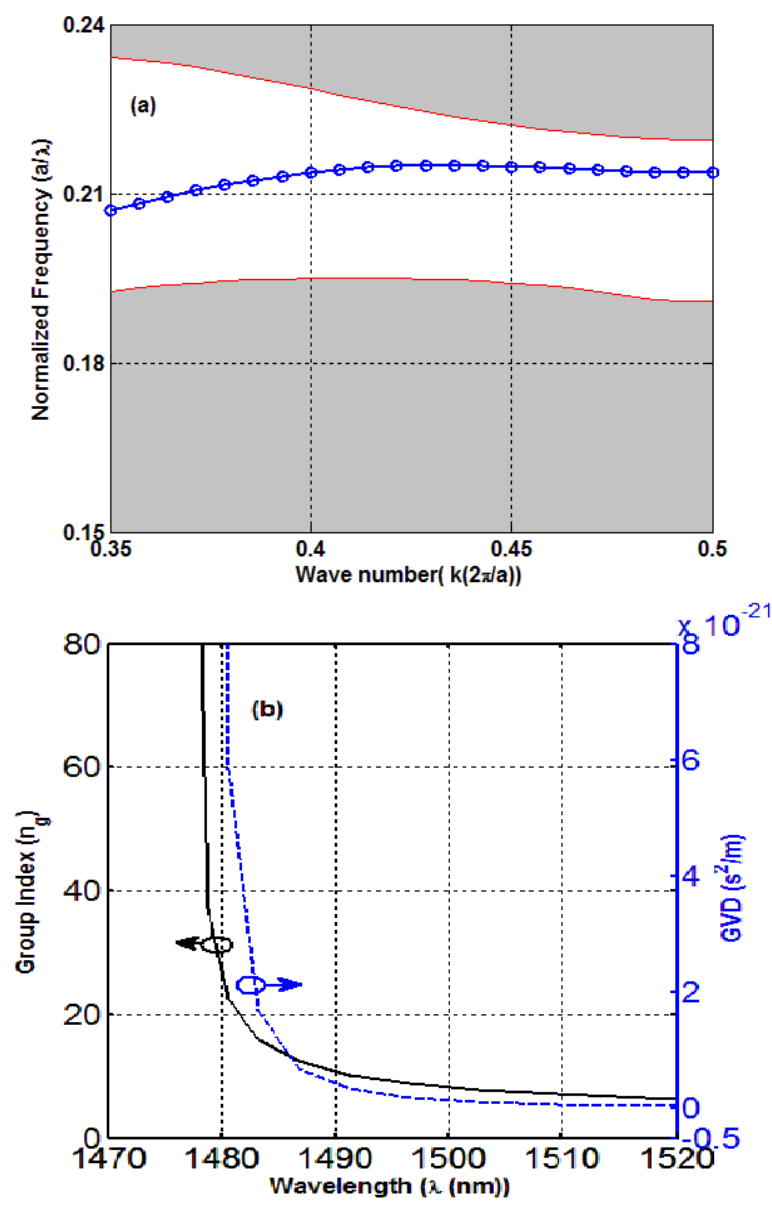

FIG. 2. (a) Calculated dispersion diagram of the fundamental mode in a slotted PCW, (b) group index and group velocity dispersion (GVD) spectra of the infiltrated slotted PCW. 

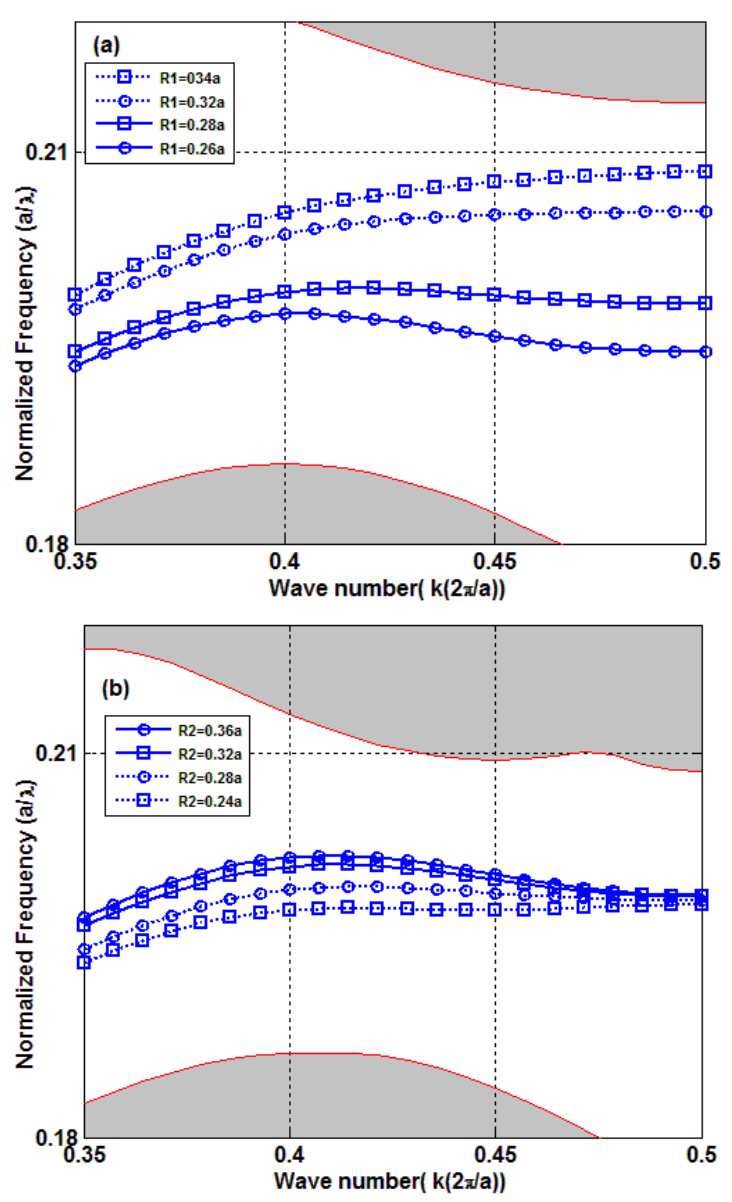

FIG. 3. The group index dependence to the radii of the two innermost rows of the holes (R1 and R2 in FIG. 1).The higher R1 will increase the tail of the diagram near band edge (a) whereas, higher R2 will increase the diagram at middle of the band (b). There must be a tradeoff between R1, R2, $n_{g}$ and flat bandwidth.

of the two innermost rows of the holes is illustrated in FIG. 3. The higher R1 will increase the tail of the diagram near band edge (FIG. 3a), whereas the higher R2 will increase the diagram at the middle of the band (FIG. 3b).

Modification of R1 and R2 will affect the available group index and its flatness over a frequency bandwidth. To achieve the slow light with large group index, $n_{g}$ over a wide bandwidth and low GVD, the modification of R1 and R2 should be combined. The optimum values of R1 and R2 can be chosen considering desired values for $n_{g}$ and flatness. Figures to FIGs. $4 \mathrm{a}$ and $4 \mathrm{~b}$ illustrate the group index and GVD for two sets of R1 and R2. The achieved group index and GVD are almost the same as those of $[12,19]$, whereas our proposed scheme stabilizes the group index of the desired structure with temperature variation.

To enable comparison between results, we have calculated the group index-bandwidth products (GBP), $n_{g}(\Delta \omega / \omega)$. The $n_{g}$ is considered as constant within a $\pm 10 \%$ range.
TABLE 1. Group index-bandwidth product $n_{g}(\Delta \omega / \omega)$ of the proposed structure at $25^{\circ} \mathrm{C}$ for different radii of two innermost rows of the holes.

\begin{tabular}{ccccc}
\hline \hline $\mathrm{R} 1 / a$ & $\mathrm{R} 2 / a$ & $n_{g}$ & $\Delta \lambda$ & $n_{g}(\Delta \omega / \omega)$ \\
\hline 0.30 & 0.30 & 40 & 0.5 & 0.01 \\
0.32 & 0.26 & 40 & 10 & 0.25 \\
0.34 & 0.26 & 24 & 16 & 0.25 \\
0.34 & 0.24 & 17 & 15 & 0.16 \\
\hline
\end{tabular}

Table 1 shows better GBP in comparison with [20] and complies very well with those of $[8,19]$.

Also, Table 1 shows that a small change in the R1 and R2 values has little effect on the GBP. Therefore the $0.02 \mathrm{a} \approx 8$ $\mathrm{nm}$ fabrication precision is acceptable.

\section{TEMPERATURE STABILITY}

Temperature dependence of the material refractive index can change the transmission behavior of the optical devices. Meanwhile when the slow light phenomenon is utilized to improve the light-matter interaction, thermo-optic effects will be enhanced $[1,8]$, hence accurate temperature control must be considered in order to guarantee the stable performance of the device for a desired temperature range. Krauss et al. showed that the temperature variation of $38^{\circ} \mathrm{C}$ can enable switching in a pure silicon based photonic crystal switch $[15,16]$. New compensation method for temperature sensitivity introduced by Chung et al. [24] and Lee et al. proposed the concept of controling temperature dependence of the properties of the slotted ring resonator devices [21]. Stabilization of optofluidic photonic crystal cavities has been demonstrated [25]. Here we show that if the suitable material is chosen and the structure is engineered properly, our proposed structure will be stable for temperature variation of up to $60^{\circ} \mathrm{C}$. Different sign of thermo-optic coefficients of the silicon and the polymer can compensate the refractive index variation and prevent undesired change in the structural behavior via thermal effects. FIG. 5 shows the structure group index for two sets of R1 and R2 of FIGs. $4 \mathrm{a}$ and $4 \mathrm{~b}$ for $\lambda=1550 \mathrm{~nm}$ and $\lambda=1530 \mathrm{~nm}$, respectively. The group index of the proposed structure varies negligibly with temperature in the range of $25^{\circ} \mathrm{C}$ to $85^{\circ} \mathrm{C}$. The propagation losses of the LFR-series polymers are less than $0.1 \mathrm{~dB} / \mathrm{cm}$ and also have thermo-optic coefficients about $-1.5 \times 10^{-4} /{ }^{\circ} \mathrm{C}$. The thermo-optic coefficient (TOC) of silicon has been assumed to be $1.8 \times 10^{-4} /{ }^{\circ} \mathrm{C}$ [16]. The group index of the structure with the same holes and slot filled by polymer, without using negative TOC of polymer for temperature compensation in the desired structure has been plotted for comparison in the same figure.

The group index-bandwidth product has been calculated at $85^{\circ} \mathrm{C}$ and depicted in Table 2. The comparison of the GBP 

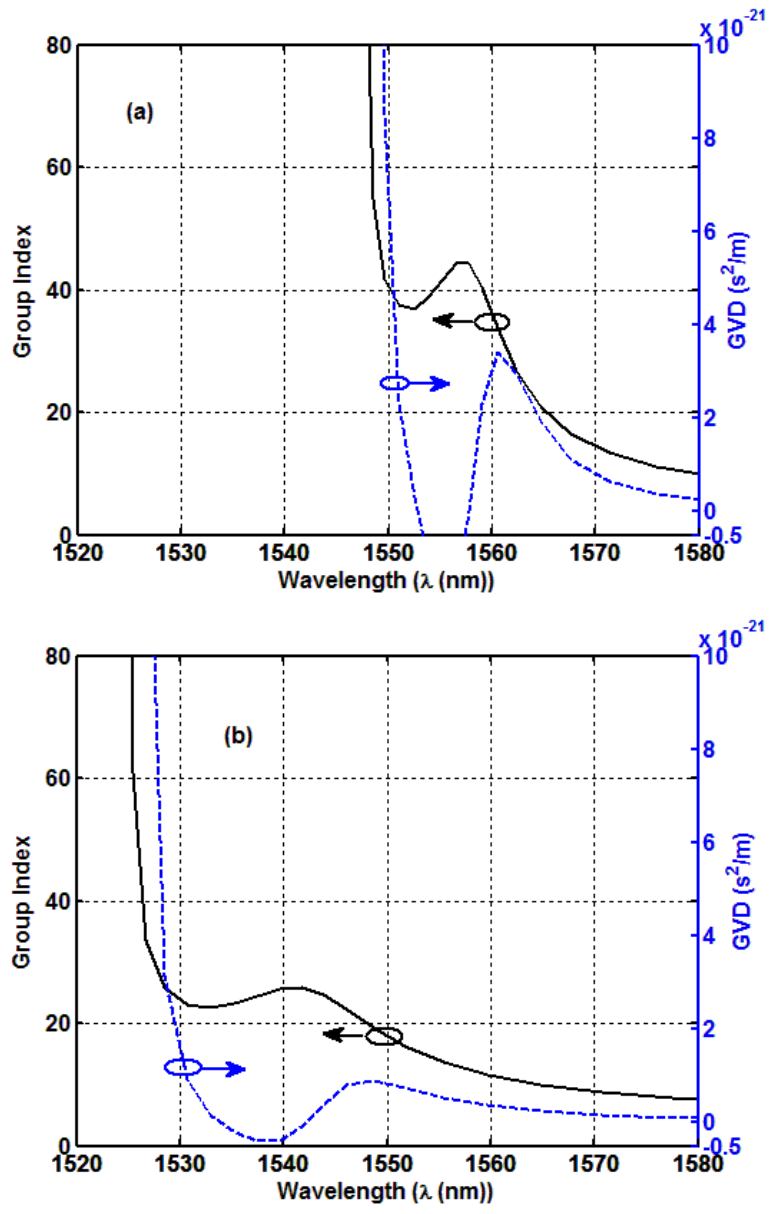

FIG. 4. Group Index and GVD of the designed sturucture for (a) R1 $=0.34 a, \mathrm{R} 2=0.26 a$ and (b) R1 $=0.32 a, \mathrm{R} 2=0.26 a$.

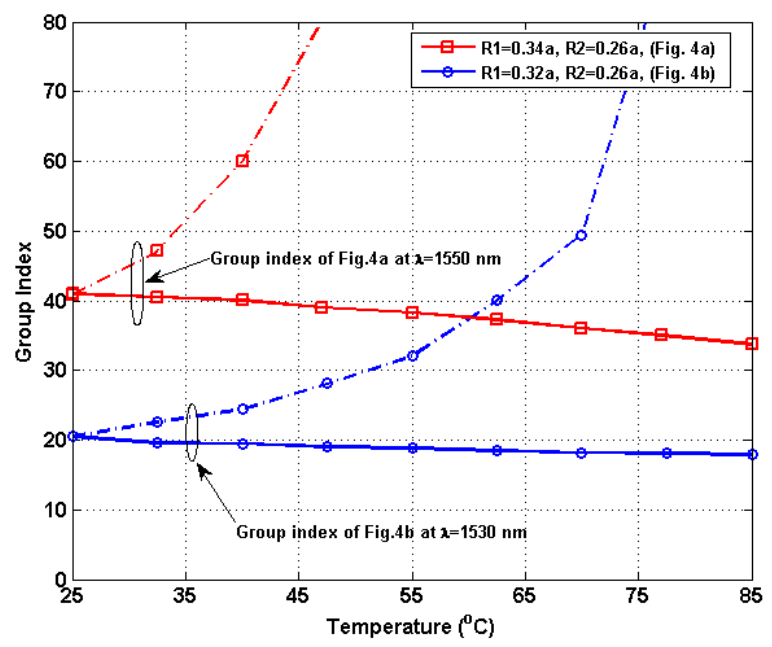

FIG. 5. Group index for two sets of R1 and R2 of Figs. 4a and $4 \mathrm{~b}$ for $\lambda=1550 \mathrm{~nm}$ and $\lambda=1530 \mathrm{~nm}$, respectively vs. temperature (solid line). The group index of the structures without using negative TOC of Polymer for compensasion are plotted for comparison (dashed line).

in $85^{\circ} \mathrm{C}$ and $25^{\circ} \mathrm{C}$ (Tables 1 and 2) shows an improvement
TABLE 2. Group index-bandwidth product $n_{g}(\Delta \omega / \omega)$ of the proposed structure at $85^{\circ} \mathrm{C}$ for different radii of two innermost rows of the holes.

\begin{tabular}{ccccc}
\hline \hline $\mathrm{R} 1 / a$ & $\mathrm{R} 2 / a$ & $n_{g}$ & $\Delta \lambda$ & $n_{g}(\Delta \omega / \omega)$ \\
\hline 0.30 & 0.30 & 40 & 0.5 & 0.01 \\
0.32 & 0.26 & 34 & 12 & 0.27 \\
0.34 & 0.26 & 22 & 22 & 0.31 \\
0.34 & 0.24 & 16 & 17 & 0.17 \\
\hline
\end{tabular}

in GBP while the group index decreases.

\section{CONCLUSION}

A new concept for design of a slow light structure based on silicon photonic crystal waveguides (PCW) has been introduced. Dispersion engineering of photonic crystal waveguide is feasible by changing the refractive index of the two innermost rows of the holes in W1 waveguide. Easy fabrication because of no requirement for high precision technology and elimination of thermal dependence of the group index on undesired temperature variation are the main advantages of this structure. The structure parameters were modified to maximize the broadband slow light effect. The accurate design and different sign of the thermo-optic coefficients of the polymer and the silicon make the proposed structure very stable in the range of $60^{\circ} \mathrm{C}$ and improve the group index-bandwidth product.

\section{REFERENCES}

1. T. Baba, "Slow light in photonic crystals," Nature 2, 465-473 (2008).

2. T. F. Krauss, "Slow light in photonic crystal waveguides," J. Phys. D: Appl. Phys. 40, 2666-2670 (2007).

3. T. Baba and D. Mori, "Slow light engineering in photonic crystals," J. Phys. D: Appl. Phys. 40, 2659-2665 (2007).

4. M. Soljacic, S. G. Johnson, S. Fan, M. Ibanescu, E. Ippen, and J. D. Joannopoulos, "Photonic-crystal slow-light enhancement of nonlinear phase sensitivity," J. Opt. Soc. Am. B 19, 2052-2059 (2002).

5. T. F. Krauss, "Why do we need slow light," Nature Photon. 2, 448-450 (2008).

6. M. Soljacic and J. D. Joannopoulos, "Enhancement of nonlinear effects using photonic crystals," Nature Materials 3, 211-219 (2004).

7. A. Y. Petrov and M. Eich, "Zero dispersion at small group velocities in photonic crystal waveguides," Appl. Phys. Lett. 85, 4866-4868 (2004).

8. L. Juntao, T. P.White, L. O'Faolain, A. Gomez-Iglesias, and T. F. Krauss, "Systematic design of flat band slow light in photonic crystal waveguides," Opt. Express 16, 6227-6232 (2008).

9. J. M. Brosi, C. Koos, L. C. Andreani, M. Waldow, J. Leuthold, and W. Freude, "High-speed low-voltage electro-optic modulator 
with a polymer-infiltrated silicon photonic crystal waveguide," Opt. Express 16, 4177-4191 (2008).

10. T. Baehr-Jones, B. Penkov, J. Huang, P. Sullivan, J. Davies, J. Takayesu, J. Luo, T. D. Kim, L. Dalton, and A. Jen, "Nonlinear polymer-clad silicon slot waveguide modulator with a half wave voltage of 0.25 V," Appl. Phys. Lett. 92, 92-94 (2008).

11. J. H. Wülbern, A. Petrov, and M. Eich, "Electro-optical modulator in a polymer infiltrated silicon slotted photonic crystal waveguide heterostructure resonator," Opt. Express 17, 304-313 (2009).

12. J. Wu, Y. Li, C. Peng, and Z. Wang, "Numerical demonstration of slow light tuning in slotted photonic crystal waveguide using microfluidic infiltration," Opt. Comm. 284, 2149-2152 (2011).

13. F. Y. M. Chan, M. J. Kim, and B. H. Lee, "Add/drop filter for CWDM systems using side-coupled long-period fiber gratings,” J. Opt. Soc. Korea 9, 135-139 (2005).

14. W. C. Kim and D. W. Park, "Analysis of temperature effects on raman silicon photonic devices," J. Opt. Soc. Korea 12, 288-297 (2008).

15. D. M. Beggs, T. P. White, L. O'Faolain, and T. F. Krauss, "Ultra compact and low-power optical switch based on silicon photonic crystals," Opt. Lett. 33, 147-149 (2008).

16. D. M. Beggs, T. P. White, L. Cairns, L. O'Faolain, and T. F. Krauss, "Ultrashort photonic crystal optical switch actuated by microheater," IEEE Photon. Technol. Lett. 21, 24-26 (2009).
17. M. Ebnali-Heidari, C. Grillet, C. Monat, and B. J. Eggleton, "Dispersion engineering of slow light photonic crystal waveguides using microfluidic infiltration," Opt. Express 17, 16281635 (2009).

18. http://ab-initio.mit.edu/wiki/index.php/MIT_Photonic_Bands.

19. J. Wu, Y. Li, C. Peng, and Z. Wang, "Wideband and low dispersion slow light in slotted photonic crystal waveguide," Opt. Comm. 283, 2815-2819 (2010).

20. S. Kubo, D. Mori, and T. Baba, "Low-group-velocity and low-dispersion slow light in photonic crystal waveguides," Opt. Lett. 32, 2981-2983 (2007).

21. J. M. Lee, D. J. Kim, G. H. Kim, O. K. Kwon, K. J. Kim, and G. Kim, "Controlling temperature dependence of silicon waveguide using slot structure," Opt. Express 16, 1645-1652 (2008).

22. M. Kim, J. J. Ju, S. K. Park, J. Y. Shim, and M. H. Lee, "Control of linear chirps in waveguide bragg gratings by applying designed core profiles," IEEE J. Lightwave Technol. 27, 4809-4813 (2009).

23. http://www.chemoptics.co.kr/.

24. Y. Chung, J. Song, W. Han, and U. Paek, "New compensation method for temperature sensitivity of fiber Brags grating using bi-metal," J. Opt. Soc. Korea 7, 84-88 (2003).

25. C. Karnutsch, C. L. C. Smith, A. Graham, S. Tomljenovic-Hanic, R. C. McPhedran, B. J. Eggleton, L. O'Faolain, T. F. Krauss, S. Xiao, and N. A. Mortensen, "Temperature stabilization of optofluidic photonic crystal cavities," Appl. Phys. Lett. 94, 231114-1 231114-3 (2009). 